\title{
Classifying Ancient West Mexican Ceramic Figures Using Three-Dimensional Modelling and Machine Learning
}

\author{
Julia Forrester-Sellers \\ Department of \\ Anthropology \\ University of Tulsa \\ 800 S College Ave \\ Tulsa, Oklahoma 74104 \\ USA \\ julie-forrester- \\ sellers@utulsa.edu
}

\author{
Alfredo Velasco \\ Tandy School of Computer Sciences \\ University of Tulsa \\ 800 S College Ave \\ Tulsa, Oklahoma 74104 \\ USA \\ alv657@utulsa.edu
}

\author{
Robert Pickering \\ Department of \\ Anthropology \\ University of Tulsa \\ 800 S College Ave \\ Tulsa, Oklahoma 74104 \\ USA \\ bob-pickering@utulsa.edu
}

\author{
J C Diaz \\ Tandy School of Computer \\ Sciences \\ University of Tulsa \\ 800 S College Ave \\ Tulsa, Oklahoma 74104 \\ USA \\ diaz@utulsa.edu
}

This work involves the creation of a taxonomy of three-dimensional posture and gesture combinations represented by ceramic human figures associated with shaft and chamber tombs in the western coastal states of Nayarit, Jalisco, Michoacán and Colima of Mexico. Gordon Hewes (1966) proposed that posture and gesture of actual human subjects communicates a tremendous amount of information that was not being gathered on a consistent basis (Hewes 1966:106). While Hewes presented his paper on "The Domain Posture" fifty years ago, his observation rings true today. The three-dimensional taxonomy should demonstrate that posture and gesture are correlated with sexual characteristics and are not random, but rather, an illustration of a purposeful series of postures and gestures that transmit cultural meaning within the ancient constructs of West Mexican culture. There is an opportunity for anthropologists to begin seeing and evaluating and documenting the non-verbal communications made through posture and gesture both in artefacts crafted as figures, as in the case of the shaft tomb figures, and in modern cultural anthropology.

The process of classification of the ceramic human figures according to posture and gesture takes three major steps: three-dimensional imaging, posture representation and finally classifying these representations. The three-dimensional imaging consists of creating three-dimensional models of the ceramic human figures. The posture representation consists of collecting measures of the fourteen major limb joints of the human body as they are represented in three-dimensional models of the ceramic figures. The last step is to use learning algorithms to classify the posture representation of the ceramic figures. The goal is to demonstrate the usefulness of the threedimensional imaging to accurately and consistently identify key posture/gesture combinations for comparison with other biological and cultural variables expressed by the figures.

Initially, this project focuses on archaeological material located in the Gilcrease Museum collection in Tulsa, Oklahoma, but will also utilise other collections as points of comparison. While this work will identify patterns of posture and gesture within West Mexican ceramic shaft tomb figures, its application does not end there. With the utilisation of technology in the form of three-dimensional imaging, its application spans both archaeological material from other cultures and modern cultural anthropology. This technology can advance studies related to the cultural meaning of this important class of artefacts. From a musicological perspective, this technology could become a critical element in metadata collection to document collections.

3D Imaging. Applications. Archaeology. Collections. Cultural heritage. Interdisciplinary. Museums. Paper. Technology.

\section{INTRODUCTION}

The information rich West Mexican shaft tomb figures have been studied by a variety of scholars with a major focus given to the dress and adornment of the figures. In fact, art historians and art dealers began in the 1940s to use empirical evidence to classify the regional styles of the shaft 
tomb figures. A great deal of attention was given to classifying the figures by style. The style classification references the West Mexican states of Nayarit, Jalisco, Colima, and Michoacán where these figures were found in controlled excavations. Official and clandestine excavations over the last four decades have resulted in the naming of additional styles and sub-styles These classifications are generally based on figure morphology, especially the facial features, colour palette and to a lesser extent clay, slip, and paint combinations.

While much focus has been on classifying the shaft tomb figures by type, very little focus has been given to identifying patterns within the shaft tomb figures by posture and gesture. This is not surprising and may be because the field of anthropology has paid scant attention to the analysis of posture and gesture. Hewes observed in 1966 that "postural terminologies have seldom been elicited by linguistics or ethnographers, and scholarly material relating to postural habits is scattered over a dozen different disciplines, and for all practical purposes has never been assembled for scientific analysis." (Hewes 1966).

This purpose of this work is to use modern technology to begin to examine issues first discussed over fifty years ago to enable anthropologists to identify and create a usable three-dimensional taxonomy of posture and gesture. Hewes saw the gap and the opportunity for posture and gesture to be recognised as a rich form of non-verbal communication, but he struggled with the lack of technology to organise his research and the lack of consistent means in which to describe the postures and gestures he recognised. Hewes identified, in 1966, the need for threedimensional analysis and evaluation of the postures and gestures.

\begin{abstract}
"[h]uman anatomy whether at the gross or microscopic level also still depends to a large extent on realistic representations of structures. The recent development of computers capable of both display and analysis of complex graphics suggests that the communicative power of pictures has not be entirely superseded by numerical representation." (Hewes 1966).
\end{abstract}

The goal of this work is to demonstrate the usefulness of the three-dimensional imaging to accurately and consistently identify key posture/gesture combinations for comparison with other biological and cultural variables expressed by the ceramic figures.

The process of classification of the ceramic human figures according to posture and gesture takes three major steps: three-dimensional imaging, posture representation and finally classifying these representations. The three-dimensional imaging consists of creating three-dimensional models of the ceramic human figures. The posture representation consists of collecting measures of the fourteen major limb joints of the human body as they are represented in three-dimensional models of the ceramic figures. The last step is to use learning algorithms to classify the posture representation of the ceramic figures.

The present work is based on observations made on the archaeological collections of the Gilcrease Museum in Tulsa, Oklahoma. Other museum collections will be used as points of comparison.

This work focus on identifying patterns of posture and gesture within West Mexican ceramic shaft tomb figures. However, its application does not end there. Utilising three-dimensional imaging technology, the process could span to both archaeological material from other cultures and modern cultural anthropology. This technology can advance studies related to the cultural meaning of this important class of artifacts. This technology could become a critical element in the metadata to document museum collections.

\section{READING NON-VERBAL COMMUNICATIONS}

\subsection{Hewes Postural Analysis}

Hewes theorised that there are universal male and female postures and gestures across cultures. (Hewes 1955). Hewes cites Boas for the proposition that "[c]ertain motor habits . . . may be stable over long periods." (Hewes 1955). Habits presented in posture could be utilised to analyse past culture contacts. He proposed an extensive 2D typology to classify the different postures. (Hewes 1966). Figure 1, provides a sequence of postures in Hewes typology. Hewes relied on published photographic evidence in his research due to the fact that ethnographic studies are scarce in detail on the points of posture and gesture. (Hewes 1955).
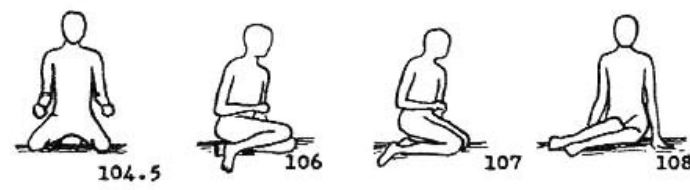

Figure 1: Postures from Hewes's typology.

\subsection{Posture correlations with biological markers}

Although formal methods of communication such as writing can be understood through translation, informal non-verbal communication like posture and gesture may be extremely difficult to decipher for outsiders although the meanings would be clear 
to those within the culture. The posture/gesture combination in Figure 1 are typical female postures. Putting aside Hewes pan-cultural hypothesis, this work looks to determine if certain posture/gesture combinations are consistently and reliably correlated with gender and other biological variables such as age.

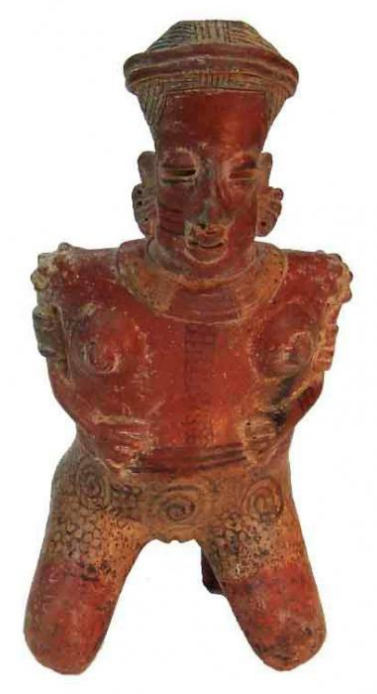

Figure 2: Gilcrease Museum, Tulsa, Oklahoma; Accession No. 54.4051.

For example, Figure 2 reflects a posture present amongst many of the West Mexican ceramic shaft tomb figures. Hewes specifically points out that "females in much of . . . Africa, Mexico, and parts of Indonesia" are found in the position described as a "deep kneel" by Hewes "with the feet under the buttocks, dorsal surfaces down". (Hewes 1955).

The postural typology displayed in Figure 3, are some of the postures that are frequently observed in the West Mexican ceramic female figures.

\footnotetext{
“. . . a strongly feminine custom, found in the American Plains and Southwest, Melanesia, South America, and southern Africa, and where there are no chairs or benches available - in Euroamerican culture." (Hewes 1955).
}

Several patterns have been identified. For example, the posture and gesture combination displayed in the example of Figure 3 is consistently female. As this work is pursued, more information should be gleaned from the postures and gestures of the West Mexican shaft tomb. An extensive discussion of the variables collected on anthropomorphic figures from west Mexico can be found in (Pickering \& Smallwood-Roberts 2014).

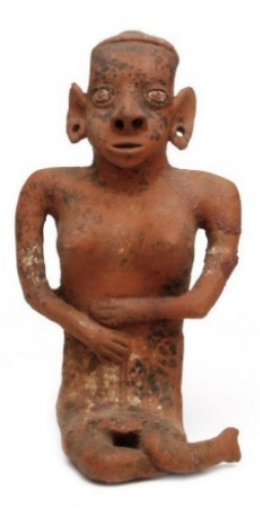

Figure 3: Gilcrease Museum, Tulsa, Oklahoma; Accession No. 54.3892.

\section{3-D MODELING AND MACHINE LEARNING}

The process of classification of the ceramic human figures according to posture and gesture takes three major steps: 3-D imaging, posture representation, and classification of these representations. The 3-D imaging consists of scanning ceramic human figures and then creating digital 3-D models. The posture representation consists of collecting measures of the fourteen major limb joints of the human body as they are represented in three-dimensional models of the ceramic figures. The last step is to use learning algorithms to classify the posture/gesture combinations of the ceramic figures.

\subsection{3-D imaging}

The estimation of human pose has been wellstudied problem in computer vision. In fact, the Kinect has the ability to reliably estimate the pose of a human user in real-time. (Kinect 2017). The Kinect skeletal tracking system works with depth images and uses a conditional random forest learning algorithm. (Kohli \& Shotton 2015).

Various issues prevented the use of this device. The skeletal system on the Kinect requires a moving target. The Kinect system expects a human sized object not a smaller ceramic figure. And the Kinect software SDK would not allow modification of these two constraints.

Intel produces now the RealSense ${ }^{\mathrm{TM}} \mathrm{R} 200$ camera. (Intel 2017). Intel RealSense SDK includes 3D scanning for stationary objects. Further, since the scanner is designed to be used on a laptop, it expects smaller objects. 


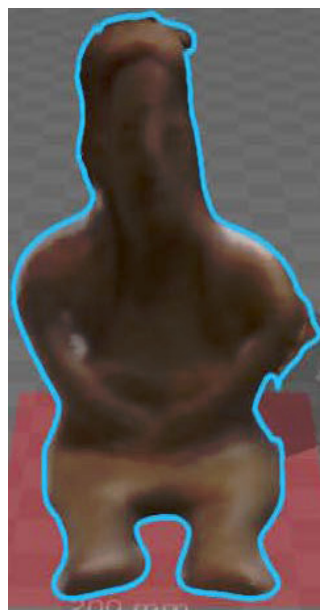

Figure 4: Scan of Figure 1000-142-126(57).

This 3D scanning can produce files that can be imported in Unity. See Figure 5.

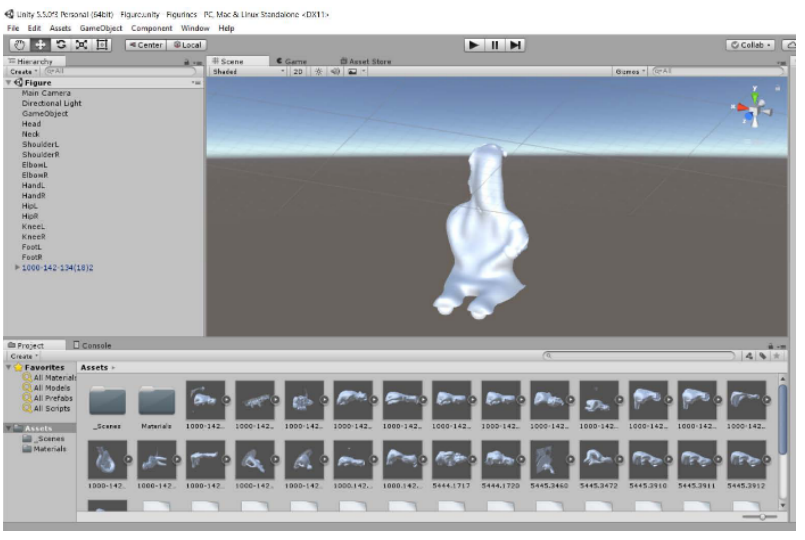

Figure 5: Unity Model of Figure 1000-142-126(57).

\subsection{Posture representation}

The joints of the figure is determined by locating their position on the 3D scan.

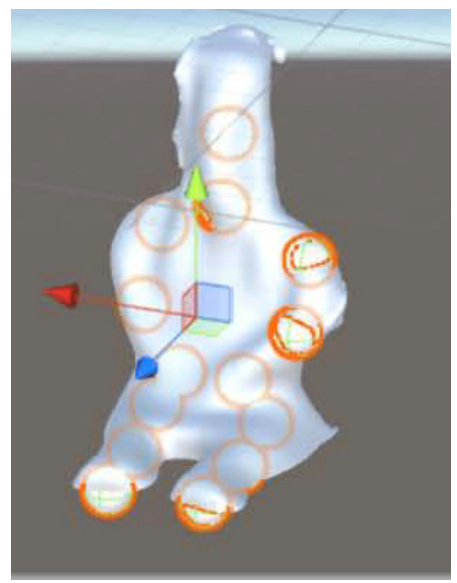

Figure 6: Position of joints for Figure 1000-142-126(57).

Then these positions are processed to obtain a vector providing the relative location of the joints with respect to other joints. This vector is normalised to unity and represented using both Cartesian and spherical coordinates $(x, y, z, \theta, \varphi)$ where the vector $(x, y, z)$ has radial unit distance $r$ $=1$, polar angle $\theta$ and azimuthal angle $\varphi$. See Figure 3.

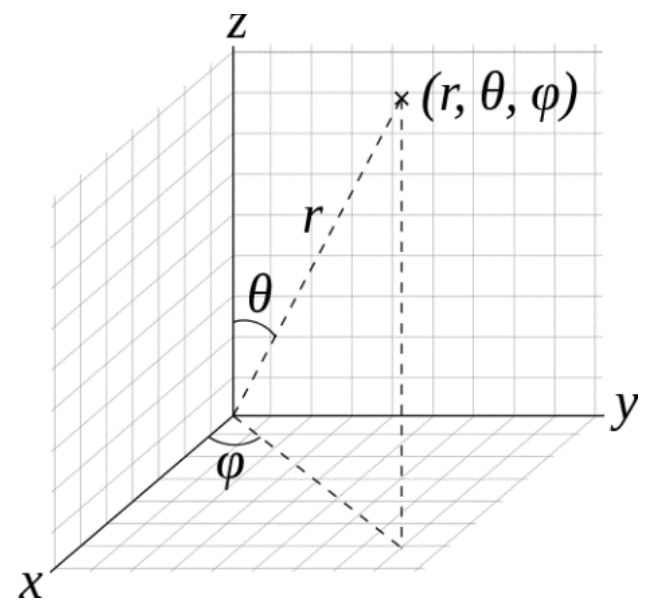

Figure 7: Spherical coordinates.

The posture representation for a given figure consist of the spherical coordinates for 14 major joints in the body: neck, shoulders, elbows, wrist, hips, knees, ankles and head position. This representation is independent of the length of the joints and at the time of this writing it does not allow for intersection of limbs such as hands on knees.

\subsection{Learning algorithms}

Decision Trees (DTs) were used to classify the position of each figure. DTs are a non-parametric supervised learning method. It works by creating a model used to predict the value of a target variable by learning simple decision rules inferred from the data features. (Scikit-learn.org 2017).

\subsubsection{Representation of Training Set}

The process is illustrated bellow with a simplified classification. The testing experiment consisted of four postures to be identified, all sitting on the floor:

- Type 1: Hands near the feet,

- Type 2: Hands near the knees,

- Type 3: Hands on the side,

- Type 4: Hands out in front

These postures were represented in Spherical coordinates which were used as a training set for DT learning algorithm.

\subsubsection{Preliminary experiment}

To illustrate the procedure for this paper, four shaft tomb figures were selected from the West Mexican Pottery Collection at the Special Collections Department. McFarlin Library, University of Tulsa, Tulsa, Oklahoma. 


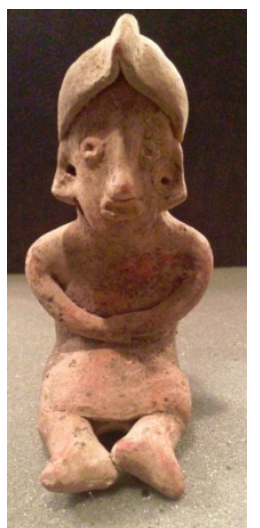

Figure 6: Shaft Tomb Figure 1000.142.84.

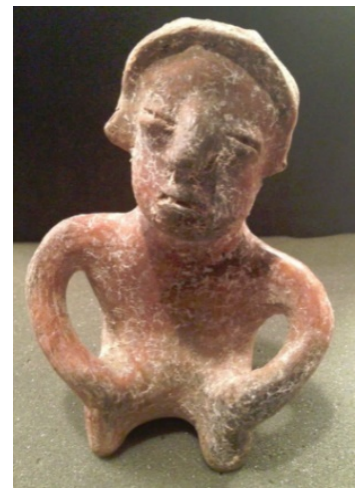

Figure 7: Shaft Tomb Figure 1000-142-134(18).

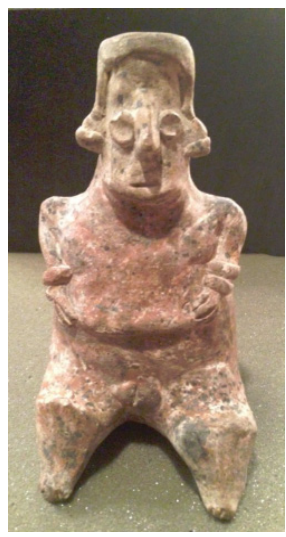

Figure 8: Shaft Tomb Figure 1000-142-134(18).

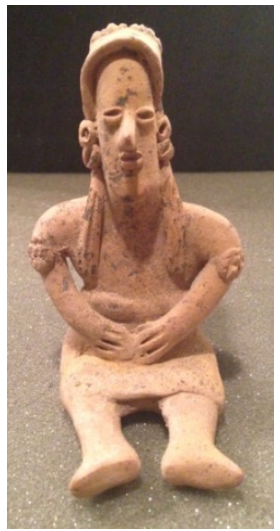

Figure 9: Shaft Tomb Figure 1000-142-126(57).

\subsubsection{Illustrative results from DT classifier}

The DT classifier was asked to recommend two ordered selections from the types in the training set. Here are illustrative results:

- $\quad$ Figure 6: Classified as Types 4, 2.

- Figure 7: Classified as Types 4, 3.

- Figure 8: Classified as Types 2, 1.

- $\quad$ Figure 9: Classified as Types 1, 2.

\subsubsection{Preliminary interpretation}

The classifier is able to classify the shaft tomb figure into some classes. Its accuracy if limited by the limited training set designed for this experiment. Significant is the limitation of the current posture representation which does not allow for intersection of limbs. The current representation is length independent to allow the identification of different size figures showing the same posture as belonging to the same class.

\section{FURTHER WORK}

There are several venues for continuing work on the learning algorithms. Constraints on the posture representation of the figures need to be added to allow representation of intersection of limbs. The training set needs to be expanded to include a larger and more representative number of possible postures. There exist a collection of data describing the angles of joint flexion on more than 300 hollow ceramic figures in the collection of the Hudson Museum, University of Maine at Orono. (Pickering unpublished). This data may be adapted for use in generating a training set for the learning algorithm.

Analysis and organisation of the shaft tomb figures by posture and gesture should reveal patterns related to sexual characteristics when compared with posture and gesture, as well as patterns of higher incidences of certain postures.

While this project has the potential to identify posture/gesture patterns within the West Mexican ceramic shaft tomb figures, its application does not end there. With the utilisation of technology in the form of three-dimensional imaging, its application spans both archaeological material from other cultures and modern cultural anthropology and will provide another avenue for study and organisation of prehistoric figures within museum collections.

Although many prominent museums have begun digitising their collections including figures, the usefulness of these efforts is limited without the utilisation of emerging technology. Adding threedimensional scanning of and posture/gesture pattern recognition will expand our abilities to identify meaningful combinations, and by using analogy from cultural anthropological examples, to 
improve our ability to ascribe meaning to significant posture/gesture combinations.

\section{REFERENCES}

Hewes, G. (1955) World Distribution of Postural Habits, American Anthropologist, 57:2, 231-44.

Hewes, G. (1966) Anthropological Linguistics, 8:8, Ethnoscience, A Symposium Presented at the 1966 Meeting of Central States Anthropological Society, 106-112.

Gilcrease Museum, 54.4051, Kneeling woman ceramic effigy with hollow construction in Lagunillas style, Kravis Discovery Center. 300 BCE - 300 CE, https://collections.gilcrease.org/object/544051 (retrieved 21 March 2017).

Gilcrease Museum, 54.3892, Seated female ceramic funerary effigy with hollow construction in Ixtlan del Rio style. Kravis Discovery Center, 300 BCE - 200 CE,

https://collections.gilcrease.org/object/543892 (retrieved 21 March 2017).
Unity. http://unity3d.com (retrieved 21 March 2017). Intel囚 RealSense ${ }^{\mathrm{TM}}$ R200. Camera https://software.intel.com/en-us/articles/realsenser200-camera (retrieved 21 March 2017).

Kinect. https://developer.microsoft.com/enus/windows/kinect (retrieved 21 March 2017).

Kohli, P. and Shotton, J. (2013) Key Developments in Human Pose Estimation for Kinect, Fossati, A., Gall, J., Grabner, H., Ren, X., and Konolige, K. (eds) Consumer Depth Cameras for Computer Vision, in Advances in Computer Vision and Pattern Recognition, Springer, London.

Pickering, R. B., (unpublished) Personal communication.

Pickering, R. B. and Smallwood-Roberts C. (2014) The Visual Guide to West Mexico Shaft Tomb Ceramic Figures. University of Tulsa, Tulsa, OK.

Scikit-learn.org. http://scikitlearn.org/stable/modules/tree.html\#tree (retrieved 21 March 2017). 